\title{
Comment on 'Second-Order Statistical Structure of Geomagnetic Field Reversals' by P. S. Naidu
}

\author{
TAD J. UlRYCh AND R. W. Clayton
}

Department of Geophysics and Astronomy, University of British Columbia, Vancouver, British Columbia, Canada

In a recent paper, Naidu [1975] has proposed that the reversal intervals of the geomagnetic field for the period 0-76 m.y. are not independent. In fact, the author has fitted a firstorder autoregressive moving average model to the data published by Heirtzler et al. [1968]. This conclusion, if true, is of importance because it suggests that the mechanism governing the reversals of the geomagnetic dynamo possesses a memory.

Naidu [1975] has developed his model on the basis of the exponential nature of the autocorrelation function calculated for the entire 76-m.y. data set. Implicit in this model is the assumption of stationarity during this time interval. However, Naidu [1971] himself has shown very clearly that the statistical structure of the polarity intervals underwent a marked transition about 48 m.y. ago. This fact has a pronounced effect on the computed autocorrelation function as demonstrated in Figure 1. It can clearly be seen that whereas the autocorrelation of the whole data set does exhibit an exponential form, the autocorrelation computed for the first 48-m.y. reversal history is impulsive at zero lag and demonstrates conclusively the independence of the intervals in this time

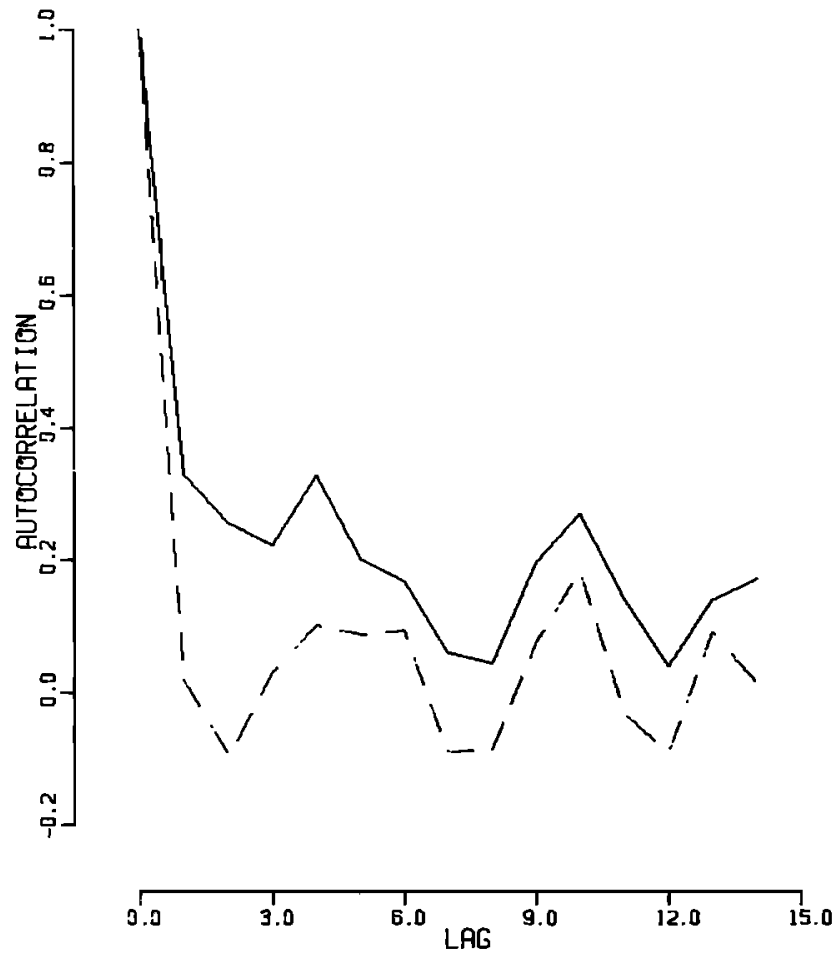

Fig. 1. Autocorrelation functions of polarity intervals. The solid line denotes the 0- to 72-m.y. segment, and the dashed line the 0- to 42m.y. segment.

Copyright $\odot 1976$ by the American Geophysical Union.

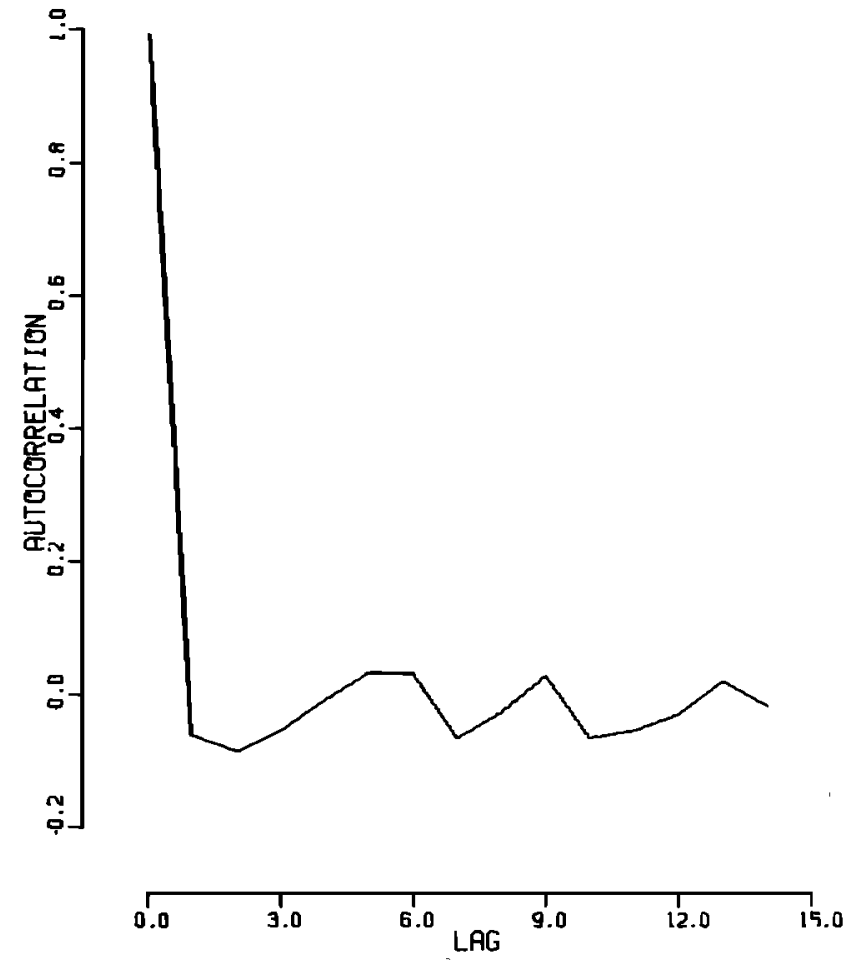

Fig. 2. Average autocorrelation function for the first six 8-m.y. segments.

span. In fact, each 8-m.y. history in the first $48 \mathrm{~m} . y$. is composed of independent intervals. The average autocorrelation of the first six 8-m.y. segments is shown in Figure 2. Meaningful second-order statistical properties of the reversals from 48 to $76 \mathrm{~m} . y$. are more difficult to determine because of paucity of data.

In summary, the reversal intervals for the first 48-m.y. history of the geomagnetic field are independent. The dependence suggested by Naidu [1975] is an artifact of the discontinuity in the statistical properties of the data at approximately $48 \mathrm{~m} . \mathrm{y}$.

\section{REFERENCES}

Heirtzler, J. R., G. O. Dickson, E. M. Herron, W. C. Pitman III, and $X$. Le Pichon, Marine magnetic anomalies, geomagnetic field reversals, and motion of the ocean floor and continents, J. Geophys. Res., 73. $2119,1968$.

Naidu, P. S., Statistical structure of geomagnetic field reversals, $J$. Geophys. Res., 76, 2649, 1971.

Naidu, P: S., Second-order statistical structure of geomagnetic field reversals, J. Geophys. Res., 80, 803, 1975.

(Received May 16, 1975; accepted August 18, 1975.) 